\title{
WHEN ARE DENSE SUBGROUPS OF LCA GROUPS CLOSED UNDER INTERSECTIONS?
}

\author{
M.A. KHAN
}

Let $G$ be a nondiscrete locally compact Hausdorff abelian group. It is shown that if $G$ contains an open torsion subgroup, then every proper dense subgroup of $G$ is contained in a maximal subgroup; while if $G$ has no open torsion subgroup, then it has a dense subgroup $D$ such that $G / D$ is algebraically isomorphic to $R$, the additive group of reals. With each $G$, containing an open torsion subgroup, we associate the least positive integer $n$ such that the nth multiple of every discontinuous character of $G$ is continuous. The following are proved equivalent for a nondiscrete locally compact abelian group $G$ :

(1) The intersection of any two dense subgroups of $G$ is dense in $G$.

(2) The intersection of all dense subgroups of $G$ is dense in $G$.

(3) $G$ contains an open torsion subgroup, and for each prime $p$ dividing the positive integer associated with $G, p G$ is either open or a proper dense subgroup of $G$.

Finally, we construct a locally compact abelian group $G$ with infinitely many dense subgroups satisfying the three equivalent conditions stated above.

\section{INTRODUCTION}

It is well-known that in nondiscrete familiar topological groups, the intersection of two proper dense subgroups need not always be dense. This paper is the result of a successful quest for those $L C A$ (that is, locally compact Hausdorff abelian) groups in which dense subgroups always have dense intersections. It is convenient to divide all nondiscrete $L C A$ groups into 2 classes:

(1) those that are torsion-open (that is, have an open torsion subgroup).

(2) those that are not torsion-open.

The two classes exhibit quite disparate behaviour as to their dense subgroups (see Theorems 2.1 and 2.2). It turns out that for any unusual, anomalous or exotic property of dense subgroups such as the one we are looking for, one must turn to torsion-open $L C A$ groups. Section 2 is devoted to them. With each nondiscrete torsion-open group, a positive integer and a finite set of primes is associated (Proposition 2.1 and Definition

Received 1st February, 1993

Copyright Clearance Centre, Inc. Serial-fee code: 0004-9729/94 \$A2.00+0.00. 
2.2). These associated integers have a crucial role in determining the behaviour of dense subgroups. Propositions 2.2 and 2.3 contain extensions of known results (see the comments preceding them). In Section 2 we prove a little more than we need. Our main result appears as Theorem 3.1. Finally we construct an $L C A$ group with an infinity of dense subgroups which are closed under arbitrary intersections (Example 3.1).

For an $L C A$ group $G, \widehat{G}$ is the group of all continuous characters, $b(G)$ the subgroup of compact elements, $t(G)$ the maximal torsion subgroup and $E(G)$ denotes a minimal divisible extension of $G$ topologised in the usual manner so that $G$ is an open subgroup. $\beta \widehat{G}=\left(G_{d}\right)^{\wedge}$ is the Bohr compactification of $\widehat{G}$. By $i: \widehat{G} \rightarrow \beta \widehat{G}$ we denote the adjoint of the identity map $G_{d} \rightarrow G$. For a subgroup $H$ of $\widehat{G}, i H$ (rather than $i(H)$ ) denotes the same group as $H$ with topology as a subspace of $\beta \hat{G}$. In particular, $i \widehat{G}$ denotes the group of all continuous characters of $G$ with topology as a subspace of $\beta \widehat{G}$, the group of all characters of $G ; i \widehat{G}$ is known to be a proper dense subgroup of $\beta \widehat{G}$, provided $G$ is nondiscrete. $\cong$ and $\approx$ denote topological and algebraic isomorphism respectively. The groups $Z(p), Z\left(p^{\infty}\right), Q, J_{p}, F_{p}$ and $T$ have their usual meanings (or see [2, page 18]). We use $R$ only as the additive reals with no topology. $\Pi$ is used for topological direct products, $\oplus$ for algebraic direct sums. $Z(p)^{m}$ and $Z(p)^{m *}$ denote the topological direct product and weak direct product of $m$ copies of $Z(p)$ respectively.

\section{TORSION-OPEN LCA GROUPS}

We begin with a definition.

Definition 2.1: Let $A$ be an abelian group and $G$ a compact torsion abelian group. $A$ is said to be $n$-bounded if $n A=0$; bounded if $n A=0$ for some $n$. $A$ is strictly $n$-bounded if $n$ is the smallest positive integer such that $n A=0$; we also say that the strict order of $A$ is $n$. $G$ is said to be trim if every open subgroup of $G$ has the same strict order as the strict order of $G . G$ is $n$-trim if $G$ is trim and strictly $n$-bounded.

The following lemma is the first step towards our characterisation of torsion-open $L C A$ groups.

Lemma 2.1. Let $G$ be a nondiscrete LCA group.

(a) If $G$ has an $m$-bounded compact open subgroup, then $\beta \widehat{G} / i \widehat{G}$ is $m$ bounded.

(b) If $\beta \widehat{G} / i \widehat{G}$ is strictly $n$-bounded, then $G$ has a compact open $n$-trim subgroup.

Proof: (a) By duality, $\widehat{G}$ has a compact open subgroup $H$ such that $m \widehat{G} \subset H$; hence also $m(i \widehat{G}) \subset i H$; since $i \widehat{G}$ is dense in $\beta \widehat{G}, m(i \widehat{G})$ is dense in $m(\beta \widehat{G})$, a 
compact subgroup of $\beta \widehat{G}$. As $m(i \widehat{G}) \subset i H$, a compact subgroup of $i \widehat{G}$, we conclude that $m(\beta \widehat{G}) \subset i H \subset i \widehat{G}$, so that $\beta \widehat{G} / i \widehat{G}$ is $m$-bounded.

(b) $n(\beta \widehat{G}) \subset i \widehat{G}$ implies that the nth multiple of every character of $G$ is continuous. Let $G(n)$ be the closed maximal $n$-bounded subgroup of $G$. It follows from $n(\beta \widehat{G}) \subset i \widehat{G}$ that the annihilators of $G_{d}(n)$ in $\beta \widehat{G}$ are the same characters (all continuous with respect to $G$ ) as the annihilators of $G(n)$ in $\widehat{G}$. Now if $G(n)$ is not open in $G$, then $G / G(n)$ has discontinuous characters also [2, 10.6]. Hence, $G(n)$ is open, so that $G$ has an $n$-bounded compact open subgroup. Using (a) and the strictness of $n$, it follows that every compact open $n$-bounded subgroup is $n$-trim.

Before our next result, we make the obvious remark that if an $L C A$ group has a compact open bounded subgroup then every compact open subgroup is bounded.

Proposition 2.1. The following are equivalent for a nondiscrete LCA group $G$ :

(a) $\beta \widehat{G} / i \widehat{G}$ is strictly $n$-bounded.

(b) $G$ has a compact open $n$-trim subgroup.

(c) $G$ contains a compact open $n$-bounded subgroup, and $n$ divides the strict order of every compact open (necessarily bounded) subgroup of $G$.

Proof: $(a) \Rightarrow(b)$ : This is Lemma 2.1 (b).

(b) $\Rightarrow$ (c): Let $H$ be a compact open $n$-trim subgroup and $H_{1}$ a strictly $r$-bounded compact open one, then $H \cap H_{1}$ is strictly $n$-bounded and also $r$-bounded. Hence, $n$ is a divisor of $r$.

(c) $\Rightarrow(a): G$ has a compact open strictly $n$-bounded subgroup $H$. By Lemma $2.1(\mathrm{a}), \beta \widehat{G} / i \widehat{G}$ is $n$-bounded. Suppose it is strictly $r$-bounded, then $r$ divides $n$. By Lemma 2.1(b), $G$ has a compact open $r$-trim subgroup, so by (c), $n$ divides $r$ and so $r=n$.

Remarks. (1) Suppose $\beta \widehat{G} / i \widehat{G}$ is strictly $n$-bounded. It is then clear from the Proposition that every $n$-bounded compact open subgroup is $n$-trim, and $n$ is the least such positive integer.

(2) Every nondiscrete torsion-open LCA group contains trim compact open subgroups (this follows from the Proposition above).

Definition 2.2: Let $G$ be a nondiscrete torsion-open $L C A$ group with a compact open $n$-trim subgroup. Let $p_{i}(i=1,2, \ldots, m)$ be the distinct primes that divide $n$. Then we shall say that $n$ is the positive integer and $p_{1}, \ldots, p_{m}$ the primes associated with $G$. (Since the strict order of $\beta \widehat{G} / i \widehat{G}$ is determined by $G$ unambiguously, the integer and primes are well-defined.) 
The next two Lemmas are related to $L C A$ groups with no open torsion subgroup.

Lемма 2.2. Let $G$ be a nondiscrete totally disconnected, $\sigma$-compact, divisible $L C A$ group such that $G=b(G)$. Then $G$ contains an algebraic direct summand isomorphic to $R$.

Proof: By [8, Corollary 6], $G \cong E(K) \times L$, where $L$ is divisible torsion and discrete and $K$ is an infinite compact power-open totally disconnected $L C A$ group. By [7, 1.1], $K \cong \prod_{p} A_{p}$, where $A_{p}$ is a topological direct product of finitely many $J_{p}$ 's and finitely many cyclic $p$-groups for each of an infinity of primes $p$. If $J_{p} \subset K$ for some $p$, then $E(K)$ contains a copy of $F_{p} \approx R$, and we are through.

Suppose now $K \cong \prod_{p} A_{p}$, where $A_{p}$ is a non-trivial finite product of discrete cyclic $p$-groups for an infinity of primes $p$. Since $K$ has cardinality $c$ and $t(K)$ is countable, the torsion-free rank of $K$ is $c$, so that $E(K)$ contains a direct summand $\approx R$.

LEMmA 2.3. Let $G$ be an LCA group which is not torsion-open. Then $G$ contains a dense subgroup $D$ such that $G / D \approx R$.

Proof: If $G$ is not totally disconnected, $G$ has a closed subgroup $H$ such that $G / H \cong T$. Now $T$ contains $R$ algebraically, so we are through. If $G$ is totally disconnected, then $G$ contains compact open subgroups none of which is a torsion group. Hence, $b(G)$, which is open in $G$, contains nondiscrete infinite cyclic subgroups, so that $b(E(G))$, which is open in $E(G)$, contains a copy of $Q$, the additive rationals with a nondiscrete topology. Since $Q$ is dense in $\bar{Q}, \bar{Q}$ is $\sigma$ compact and divisible $[1$, Theorem 2]. Let $E(G)=\bar{Q}+L$ be an internal algebraic direct sum, then $Q+L$ is dense in $E(G)$, so $D=(Q+L) \cap G$ is a proper dense subgroup of $G$. Since $G$ is open and $Q+L$ is dense in $E(G)$, we have $E(G)=G+Q+L$, so $G / D \approx(G+Q+L) /(Q+L)=E(G) /(Q+L)$ $=(\bar{Q}+L) /(Q+L) \approx \bar{Q} / Q$. Now $\bar{Q}$ is a nondiscrete totally disconnected $\sigma$-compact divisible $L C A$ group with every element compact, so by Lemma $2.2, \bar{Q}$ contains $R$ algebraically. Hence, so does $G / D$ and the proof is complete.

In the next two Theorems, we characterise separately two classes of $L C A$ groups: (i) those that are torsion-open. (ii) those that are not torsion open.

Theorem 2.1. The following are equivalent for a nondiscrete $L C A$ group $G$ :

(a) $G$ is torsion-open.

(b) Every proper dense subgroup of $G$ is contained in a maximal subgroup of $G$.

(c) $\beta \widehat{G} / i \widehat{G}$ is bounded.

Proof: $(\mathrm{a}) \Rightarrow(\mathrm{b})$ : Let $D$ be a proper dense subgroup, $\boldsymbol{H}$ a compact open $n$ bounded subgroup, then $G=D+H$, so $G / D \approx H / B \cap D$, which is $n$-bounded. clearly, $D$ is contained in a subgroup of prime index $p$ dividing $n$. 
(b) $\Rightarrow$ (a): Follows from Lemma 2.3.

(a) $\Rightarrow$ (c): Follows from Lemma 2.1 .

(c) $\Rightarrow$ (a): This is from Proposition 2.1.

For the record and for use, we next deal with groups which are not torsion-open.

TheOREM 2.2. The following are equivalent for a nondiscrete $L C A$ group $G$ :

(a) $G$ is not torsion-open.

(b) $G$ contains a dense subgroup $D$ such that $G / D \approx R$.

(c) $\beta \widehat{G}$ contains a closed subgroup $H \cong\left(R_{d}\right)^{\wedge}$ such that $H \cap i \widehat{G}$ is trivial.

Proof: (a) $\Rightarrow$ (b): Lemma 2.3.

(b) $\Rightarrow$ (a): Follows from Theorem 2.1 .

(b) $\Rightarrow$ (c): $D$ separates points of $\widehat{G}$, so $D$ annihilates no nonzero continuous characters. Now $H=A\left(\beta \widehat{G}, D_{d}\right) \cong\left(R_{d}\right)^{\wedge}$ and $H \cap i \widehat{G}$ is trivial.

(c) $\Rightarrow$ (a): Since $\beta \widehat{G} / i \widehat{G}$ is not bounded, $G$ cannot be torsion-open (Theorem 2.1).

REMARK. Let $G$ be a nondiscrete torsion-open group with associated integer $n$. Then $G$ has a compact open $n$-trim subgroup $H$. Now $H$ is divisible by every prime not a divisor of $n$. Hence $p G$ is automatically open for every prime $p$ that is prime to $n$. So there remain only a finite number of primes, namely those that divide $n$, which can possibly provide dense subgroups of prime indices. This is the main use of the associated integer $n$.

We end this section after a brief detour. Substantially, the following result appeared in [5], [6, Corollary 5.1] and [4]: A nondiscrete $L C A$ group $G$ contains no proper dense subgroup if and only if $G$ is torsion-open and $p G$ is open for each prime $p$. We refine this statement a bit and give a shorter proof.

First we prove a lemma to be used more than once.

Lemma 2.4. Let $G$ be a nondiscrete $L C A$ group with $p G=0$. Then $G$ contains both a dense maximal subgroup and an open maximal subgroup.

Proof: By [3, Theorem 25, 29] $G \cong L \times M$ where $L$ is discrete and $M$ is an infinite topological direct product, say $Z(p)^{m}$. Since the direct sum of these $Z(p)$ 's is dense in $M, G$ contains a proper dense subgroup and so a maximal one too. Clearly $G$ contains open subgroups of index $p$. (Look at the dual.)

Proposition 2.2. A nondiscrete $L C A$ group $G$ contains no proper dense subgroup if and only if $G$ is torsion-open and $p G$ is open for each associated prime $p$.

Proof: For the sufficiency, we note that by the Remark above, $p G$ is open for each prime $p$, so there are no dense maximal subgroups for proper dense subgroups to 
be contained in.

Conversely, assume there are no proper dense subgroups. Then $G$ is torsion-open (Theorem 2.2). Suppose $p G$ is nonopen for some associated prime $p$. As each maximal subgroup of index $p$ is open (otherwise it would be dense), their intersection $p G$ is closed. Hence, $G / p G$ is a nondiscrete $L C A$ group with no dense subgroup of index $p$, contradicting Lemma 2.4. Hence, $p G$ is open and the proof is complete.

In [2, 7.18], D.L. Armacost asked which $L C A$ groups contain no proper pure dense subgroups. We do not have the complete answer but Theorem 2.2. leads to the following.

PROPOSITION 2.3. If an LCA group is not torsion-open, then it contains proper pure dense subgroups.

\section{ThE MAIN RESUlts}

We begin with a couple of lemmas.

LEMMA 3.1. Let $m G$ and $n G$ be proper dense subgroups of an $L C A$ group $G$. Then $m n G$ is also dense in $G$.

Proof: We know $n: G \rightarrow G, n(g)=n g$, is a continuous endomorphism of $G$. By continuity of $m, m G=m(\overline{n G}) \subset \overline{m n G}$, so that $G=\overline{m G} \subset \overline{m n G}$.

Lemma 3.2. Let $G$ be a nondiscrete $L C A$ group and let $D$ and $H$ be respectively a proper dense subgroup and a proper open subgroup of $G$. Then $G$ contains two proper dense subgroups whose intersection is not dense in $G$ in each of the following cases:

(i) $G / D \approx G / H \approx Z(p)$ for some prime $p$.

(ii) $G / D \approx G / H \approx Z\left(p^{\infty}\right)$ for some prime $p$.

(iii) $G / D \approx Z\left(p^{\infty}\right)$ and $G / H \approx Z(p)$ for the same prime $p$.

Proof: (i) We have $G / D \cap H \approx(D / D \cap H) \oplus(H / D \cap H) \approx Z(p) \oplus Z(p)$. Let $f: G \rightarrow G / D \cap H$ be the natural map; choose a subgroup $A$ of $G$ containing $H \cap D$ such that $f(A) \approx Z(p), f(A) \cap f(D)=f(A) \cap f(H)=0$. Now $A$ is a maximal dense subgroup, for $A \cap H=D \cap H$ is not open. Hence, $A$ is dense in $G$ but $A \cap D=H \cap D$ is not dense in $G$.

(ii) Doing as in (i), we see that $G / H \cap D \approx Z\left(p^{\infty}\right) \oplus Z\left(p^{\infty}\right)$. Let $A$ be a subgroup of $G$ containing $H \cap D$ such that $f(A) \approx Z\left(p^{\infty}\right)$ and $f(A) \cap f(D)=f(A) \cap f(H)=0$. We claim that $\bar{A}=A+H=G$. Since $A \subset A+B$ which is open, $\bar{A} \subset A+B$. Also $A \subset \bar{A}, B=\overline{H \cap D} \subset \bar{A}$ implies that $A+H \subset \bar{A}$, so $\bar{A}=A+B$. Since $H$ and $A$ both contain $B \cap D$ and $f(B)$ and $f(A)$ together generate $G / H \cap D$, we must have $A+B=G$. Now $A$ is a proper dense subgroup of $G$ such that $A \cap D=H \cap D$ is not dense in $G$. 
(iii) Here $G / H \cap D \approx(D / D \cap H) \oplus(H / D \cap H) \approx Z\left(p^{\infty}\right) \oplus Z(p)$. Let $f: G \rightarrow$ $G / H \cap D$ be the natural map. Choose a subgroup $A$ containing $B \cap D$ such that $f(A) \approx Z\left(p^{\infty}\right), f(A) \cap f(B)=f(A) \cap f(D)=0$. We claim $\bar{A}=A+B=G$. Since $B$ is already a maximal subgroup of $G, A+B=G$. Since $A \subset A+B$ which is open, $\bar{A} \subset A+H$. Also $A \subset \bar{A}, H=\overline{A \cap D} \subset \bar{A}$ implies $A+B \subset \bar{A}$. Hence, $\bar{A}=A+B=G$ as claimed. Now $A$ is a proper dense subgroup of $G$ such that $A \cap D=B \cap D$ is not dense in $G$.

We now come to the main result of this paper.

THEOREM 3.1. The following conditions are equivalent for a nondiscrete $L C A$ group $G$ :

(a) $G$ is torsion-open and for each prime $p$ associated with $G, p G$ is either open or a proper dense subgroup of $G$.

(b) The intersection of all dense subgroups of $G$ is dense in $G$.

(c) The intersection of any two dense subgroups of $G$ is dense in $G$.

Proof: (a) $\Rightarrow$ (b): suppose the associated integer $n=p_{1}^{r_{1}} \ldots p_{m}^{r_{m}}$, where the $p_{i}$ 's are distinct primes. If $p_{i} G$ is open for all $i$ in question, then $G$ contains no proper dense subgroups to worry about (Proposition 2.2). Assume $p_{i} G$ is proper dense for $i$ from 1 to $k$, and open otherwise. Since $p_{1} G, \ldots, p_{k} G$ are proper dense in $G$, it follows by repeated application of Lemma 3.1 that $p_{1}^{\tau_{1}} \ldots p_{k}^{r_{k}} G$ is a proper dense subgroup of $G$. We shall show that every proper dense subgroup $D$ of $G$ contains it. Let $n_{1}=p_{1}^{r_{1}} \ldots p_{k}^{r_{k}}$. Let $H$ be a compact open $n$-trim subgroup. Since $G=H+D$, $G / D \approx H / D \cap H$ is $n$-bounded. Now a bounded abelian group contain a maximal subgroup of index $p$ if and only its $p$-component is nonzero. Since $p_{i} G$ is open for $i=k+1, \ldots, m$, it is clear that $D$ is not contained in any maximal subgroup of index $p_{i}(i=k+1, \ldots, m)$. Hence, $G / D$ is $n_{1}$-bounded that is, $n_{1} G \subset D$.

(b) $\Rightarrow$ (c): trivial.

(c) $\Rightarrow$ (a): Suppose $G$ is not torsion-open, then for each prime $p, G$ contains a dense subgroup $D_{p}$ such that $G / D_{p} \approx Z\left(p^{\infty}\right)$; this follows from Theorem 2.2. Assume first that $G$ is not totally disconnected. Then there is a closed subgroup $K$ in $G$ such that $G / K \cong T$. Since $Z\left(2^{\infty}\right)$ and $Z\left(3^{\infty}\right)$ are proper dense subgroups of $T$ with trivial intersection, $G$ must contain two proper dense subgroups with intersection $K$, contradicting (c). So $G$ is totally disconnected and contains an open subgroup $B$ such that $G / H \approx Z(q)$ or $Z\left(q^{\infty}\right)$ for some prime $q$. By Lemma 3.2, this contradicts (c). Hence $G$ must be torsion-open. Suppose finally that for some prime $p$ associated with $G, p G$ is neither open nor dense in $G$. Then $\overline{p G}$ is a proper subgroup of $G$. If every subgroup of index $p$ in $G$ were open, then $p G$ would be open (Lemma 2.4). Hence, $G$ contains a dense subgroup of index $p$. If $\overline{p G}$ is open, then $G$ contains an open 
subgroup of index $p$. If not, the same conclusion follows from Lemma 2.4. Lemma 3.2 then contradicts (c). Hence, $p G$ is either open or a proper dense subgroup of $G$. This completes the proof.

REMARKs. (1) Nondiscrete $L C A$ groups with no proper dense subgroups satisfy the conditions of Theorem 3.1 trivially. Such for example are torsion divisible $L C A$ groups.

(2) No infinite compact torsion abelian group $G$ satisfies the conditions of Theorem 3.1. For $p G$ is closed for each prime $p$. Considering $\widehat{G}$, it is clear that $p G$ is open for each associated prime if and only if $G$ is finite.

(3) By now, one may start wondering if there are any $L C A$ groups that satisfy the conditions of Theorem 3.1 nontrivially. The following example is therefore in order.

EXAMPLE 3.1. Let $H=Z(p)^{m}$ where $m$ is an infinite cardinal. Since $Z(p)^{m *}$ is dense in $H, H$ contains infinitely many dense subgroups both of finite and infinite indices in $H$. Consider a fixed $L C A$ group $E(H)$. Let $K$ be a dense subgroup of infinite index in $H$ and let $E(K)$ be a minimal divisible extension of $K$ in $E(H)$. Considering their socles (see [2, page 8]), it is clear that $E(K)$ is a proper subgroup of $E(H)$. Also $E(K)$ is nonclosed for $E(K) \cap H=K$ is nonclosed. It is easy to check that $\overline{E(K)}=E(K)+H$. Set $G=\overline{E(K)}$. Then $G$ is torsion-open, $p$ is the only prime associated with $G$ and $p G=E(K)$ is a proper dense subgroup of $G$. Let $D$ be an arbitrary proper dense subgroup of $G$. Then $G=D+H$ implies $E(K)=p G=p(D+H)=p D \subset D$. So every proper dense subgroup of $G$ contains $E(K)$. Since

$$
G / E(K)=(E(K)+H) / E(K) \approx H / H \cap E(K)=H / K,
$$

which is infinite, it follows that $G$ contains infinitely many dense subgroups each containing the dense subgroup $E(K)$.

\section{ReFerences}

[1] D.L. Armacost and W.L. Armacost, 'On Q-dense and densely divisible LCA groups', Proc. Amer. Math. Soc. 36 (1972), 301-305.

[2] D.L. Armacost, The structure of locally compact abelian groups (Marcel Dekker, Inc., New York, 1981).

[3] E. Hewitt and K.A.Ross, Abstract harmonic analysis, Vol.1 (Springer-Verlag, Berlin, Heidelberg, New York, 1963).

[4] M.I. Kabenyuk, 'Dense subgroups of locally compact abelian groups', Siberian Math. J. 21 (1980), 902-903.

[5] M.A. Khan, 'Chain conditions on subgroups of LCA groups', Notices Amer. Math. Soc. 24 (1977), p. 443.

[6] M.A. Khan, 'Chain conditions on subgroups of LCA groups', Pacific J. Math. 86 (1980), 517-534. 
[7] M.A. Khan, 'Power-rich and power-deficient LCA groups', Canad. J. Math. 33 (1981), $664-670$.

[8] M.A. Khan, 'A theorem on power-open LCA groups and its Consequences', Bull. Austral. Math. Soc. 26 (1982), 239-247.

Department of Mathematical Sciences

Umm al-Qura University

PO Box 3711

Makkah

Saudi Arabia 